\title{
The Reasons for Douyin's Success from the Perspective of Business Model, Algorithm and Functions
}

\author{
Baiyu Huang \\ School of Information Engineering, Guangdong University of Technology, Guangzhou, Guangdong Province, 51006, \\ China \\ Corresponding author's e-mail:250539415@qq.com
}

\begin{abstract}
In recent years, short video applications have sprung up in China. As one of the most successful short video applications, TikTok allows users to be fully entertained in fragmented time. Douyin is the name of the Chinese version of TikTok. The number of users has shown explosive growth, and it has set off a download frenzy at home and abroad. This article will study TikTok's business profit model from the data obtained from various platforms, user experience, software algorithms and surveys. This research shows that understanding the users' favourite recommendations based on big data algorithms, as well as seizing the rhythm of the times and the advancement of mobile technology are important for companies to get profit.
\end{abstract}

Keywords: TikTok, short video, We Media, business model analysis, survey

\section{INTRODUCTION}

With the development of the mobile Internet, the upgrading of communication technology and the Chinese government's "Internet" policy support, short video software experienced explosive development in 2016. According to QuestMobile data, as of June 2019, the number of users of short video apps exceeded 820 million, which reached a year-on-year growth rate of over $32 \%$ [1]. The time that the users spend on these apps accounted for $11.4 \%$ of the total online time [1]. The number of monthly active users of short video and Online video is further reduced, and short video applications have become the second largest application type after instant messaging [1].

TikTok is a music creative short video social software released by ByteDance, which was launched on September 20, 2016. TikTok was first developed in the Chinese market. After beating other short video applications and becoming the most active short video application in China, TikTok was launched outside China. After ByteDance acquired Musical.ly, a short video app in North America, it merged its users into its own users. Currently, TikTok is the world's most downloaded APP with 500 million users (monthly active users) worldwide.

Through the collected data, user experience reports and surveys, this article will study TikTok's business profit model, analyze the reasons for its popularity in the world and its revenue model, thus providing reference for Chinese software to go global.

\section{BACKGROUND INFORMATION OF TIKTOK}

At present, TikTok's download volume and number of users are growing exponentially. According to official data released by TikTok, TikTok has been available for download in 39 languages in more than 150 countries around the world. TikTok global active users are estimated to be 800 million, among which Chinese users account for more than $50 \%$. As of July 2020 , this social application has 100 million users in the United States, an increase of nearly $800 \%$ over January 2018 [2].

According to Sensor Tower data, as of July 2020, TikTok has been downloaded 65.2 million times, ranking first in the global non-gaming mobile application download list and revenue list [3]. The data have increased by $21.4 \%$ compared with the same period in 2019, and it has beaten Facebook and YouTube. And Instagram and other well-known apps. TikTok's revenue in 2019 reached 176.9 million US dollars (excluding noniOS China revenue) [3]. Especially during the COVID19 period, many people have to stay at home in isolation. Watching TikTok makes the fragmented time at home less boring. It is an excellent entertainment social media. 
Because of this, TikTok's download volume has also increased significantly during this period. As shown in fig. 3, as of January 2020, TikTok is ranked 7th in the list of the most frequently used social platforms in the world, and the world's first in the short video field. About $50 \%$ of TikTok's global audience are under the age of 34, and $26 \%$ of them are between 18 and 24 years old. India and the United States are one of TikTok's largest overseas markets. Statistics from the Global Network Index show that TikTok's penetration rate seems to be the highest in Asia, with a penetration rate of $36 \%$. As shown in fig. 4 , more than a third of 16-64-year-old users in the region have this account. The gap between the rest of the world is small, with penetration rates between $12 \%$ (North America) and 10\% (Latin America and Europe) [2]. TikTok is popular all over the world. Many people have gained happiness and made money from TikTok. As a result, the Indian and US governments have recently met with resistance and protests from users when they unreasonably ban TikTok. They even launched the topic of "Save TikTok" on TikTok. These macro data are enough to show that TikTok has successfully exited China and occupied a certain share in the global market. TikTok has become one of the important social media in the new era and at the same time demonstrates the role of public opinion.

\begin{tabular}{|c|c|c|c|c|}
\hline & Overall Revenue & App Store Revenue & & ogle Play Revenue \\
\hline 1 & Douyin \& TikTok & 1 J Douyin \& TikTok & 11 & Google One \\
\hline 20 & YouTube & 2 YouTube & 2 & BIGO LIVE \\
\hline 3 & Tinder & 30 Tinder & 30 & Tinder \\
\hline 40 & Tencent Video & $4 \bigcirc$ Tencent Video & 4 约 & Piccoma \\
\hline 5 & iQIYI & 5 iQIYI & $5 \Theta$ & LINE \\
\hline 6 & Disney+ & Netflix & $6 \infty$ & Disney+ \\
\hline 7 & BIGO LIVE & 7 Disney+ & 7 D & Twitch \\
\hline 8 & Netflix & 8 毞 Piccoma & 8 하뭉 & LINE Manga \\
\hline 9 枈 & Piccoma & 9 L & 9 P & Pandora \\
\hline $10 \div$ & LINE Manga & 10 BIGO LIVE & $10 f$ & Facebook \\
\hline
\end{tabular}

Figure 1 The world's top 10 popular mobile app revenue in July [3]

\begin{tabular}{|c|c|c|c|c|c|}
\hline \multicolumn{2}{|r|}{ Overall Downloads } & \multicolumn{2}{|c|}{ App Store Downloads } & \multicolumn{2}{|c|}{ Google Play Downloads } \\
\hline 1 & F Douyin \& TikTok & 1 & Douyin \& TikTok & $1 \mathrm{C}$ & Douyin \& TikTok \\
\hline 2 & Facebook & 20 & YouTube & $2 f$ & Facebook \\
\hline 3 & 0 ZOOM & $3 \sqrt{c}$ & Instagram & 30 & ZOOM \\
\hline 4 & (0) Instagram & $4 \mathrm{CO}$ & ZOOM & 4 (9) & WhatsApp \\
\hline 5 & (C) WhatsApp & 5 & WhatsApp & 5 () & Instagram \\
\hline 6 & ( Messenger & 6 & Facebook & 69 & Google Meet \\
\hline 7 & (.) Google Meet & $7 \approx$ & Messenger & $7 \approx$ & Messenger \\
\hline 8 & $D$ Snack Video & $8 ?$ & Google Maps & 80 & Snack Video \\
\hline 9 & Likee & 9 & Pinduoduo & $9 \odot$ & Likee \\
\hline 10 & (4) Telegram & $10 M$ & Gmail & 103 & Telegram \\
\hline
\end{tabular}

Figure 2 The world's top 10 popular mobile app downloads in July [3]

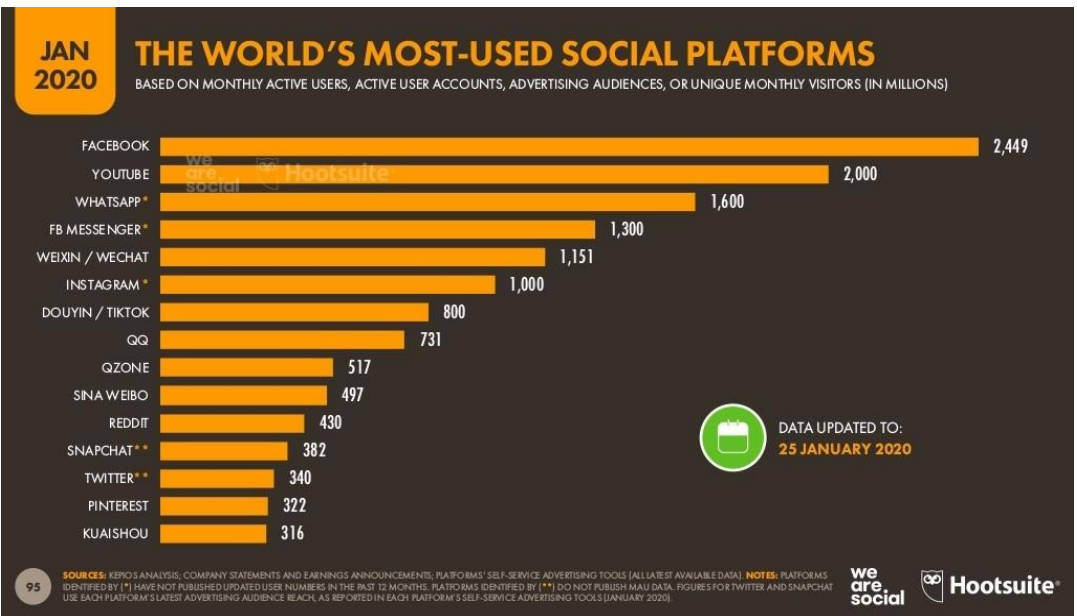

Figure 3. The world's most-used social platforms in January, 2020 [2] 


\section{TikTok Around the World}

$\%$ of internet users aged 16-64 who are members of TikTok by global region

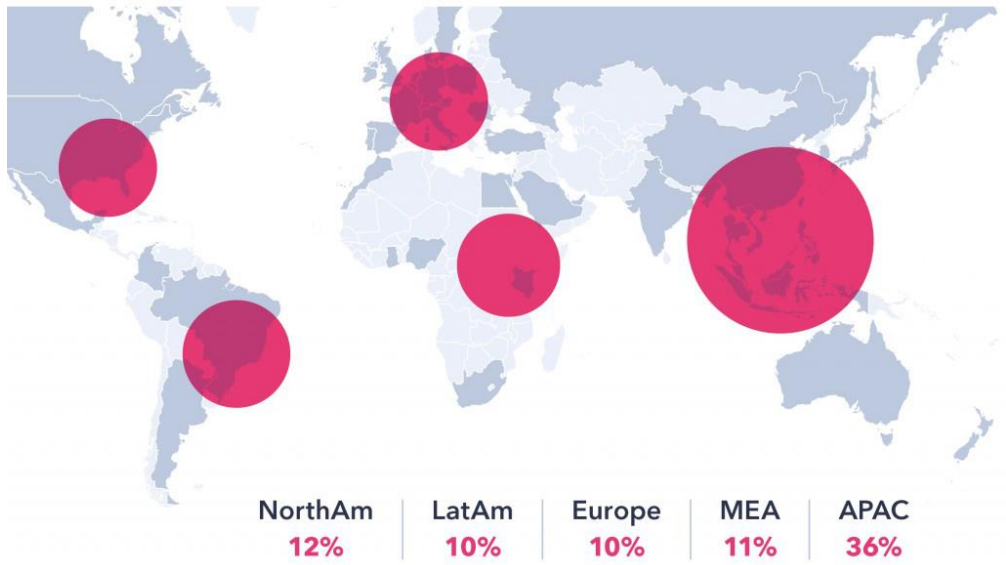

Question: On which of the following services do you have an account? TikTok

Source: GlobalWeblndex Q2 2019 Base: 139,658 internet users aged 16-64

Figure 4. TikTok around the world [2]

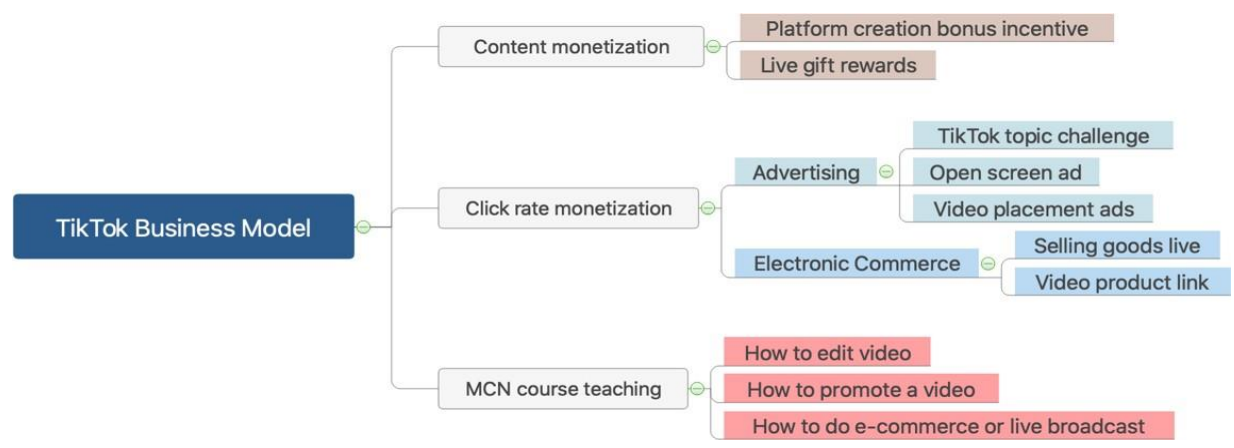

Figure 5. The framework of TikTok's business model

\section{ANALYSIS ON ITS BUSINESS MODEL}

According to fig. 5, the business profit model can be divided into three types: content monetization, clickthrough rate monetization and MCN course teaching.

\subsection{Content monetization}

First of all, TikTok's content is divided into short videos and live broadcasts. It is short videos that mainly attract users. TikTok will officially give users incentive bonuses for video creation. Video creators who have accumulated a large number of fans can open live broadcasts on the basis of video bonuses. The virtual gifts given by viewers to reward anchors are also one of the sources of profit for TikTok and creators.

\subsection{Click-through rate monetization}

Then, the click-through rate monetization means that after the video is popular among users, the creator can receive advertisements or do e-commerce. On TikTok, there are usually three forms of advertising: topical challenges and screen ads, and video placement ads.
There are two ways for e-commerce: live sales and product links in videos. Most of TikTok's business comes from this. In order to attract more users, TikTok has invited many celebrities to settle on TikTok. The presence of celebrities not only brings attention to TikTok, but also increases fans for the celebrities themselves. Merchants are also happy to cooperate with TikTok and celebrities. They can promote products in videos, or they can open live broadcasts to sell goods [5]. At the same time, TikTok has an advertising trading platform called Xingtu, which is called TikTok creator marketplace in English. Brands and creators can seek cooperation on it to form a win-win cooperation. Take the well-known Chinese actor Chen $\mathrm{He}$ as an example. He often shares interesting videos and life clips on the Chinese version TikTok, Douyin. At the same time, relying on his previous popularity, he quickly gained 70 million fans on this platform. In his life video, there will be some products he needs to promote. After accumulating a large number of fans, he often broadcasts live broadcasts for e-commerce sales. There are tens of thousands of viewers in the live broadcast room, and the transaction amount can reach tens of millions. Click- 
through rate monetization is fast, which is the charm of TikTok's business model.

\subsection{MCN course teaching}

Finally, some MCN organizations including TikTok officials will provide paid courses to teach you how to make money with TikTok. The content of the course is about how to edit the video, how to promote the video and how to do e-commerce or live broadcast [4]. The MCN agency has incubated a large number of short video creators to help them create videos and promote them, thereby accumulating a large number of fans. Then monetize the fan value of popular creators. Making money is the most important function of MCN, but this kind of paid course teaching is very necessary. This is one of the necessary steps for many ordinary people to become big influencers. At the same time, this is also an important part of TikTok's business profit model. A steady stream of creators is like new blood, providing TikTok with new creative vitality.

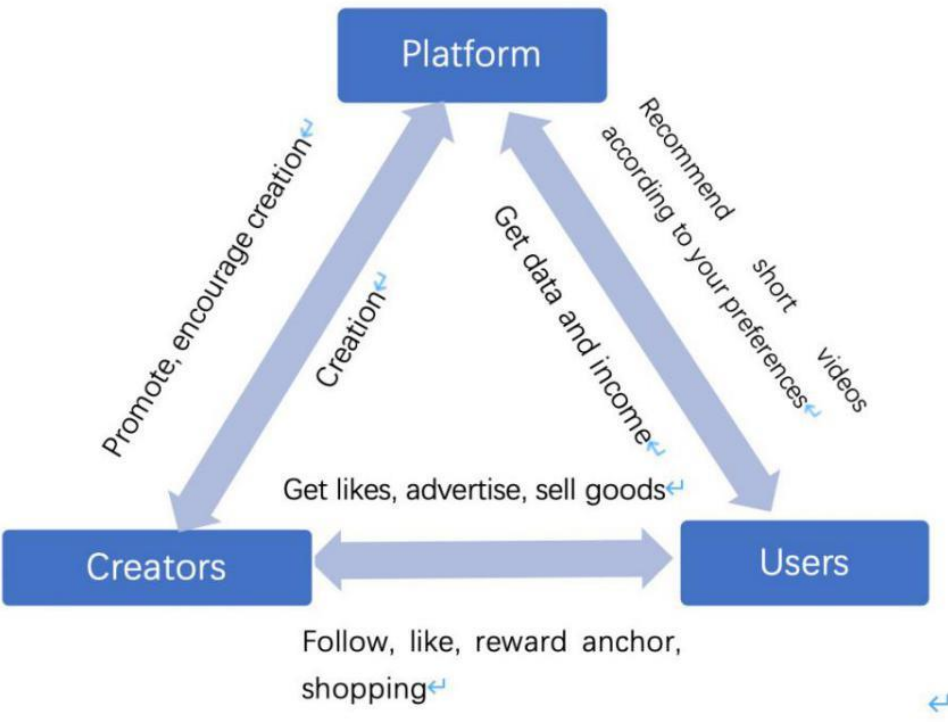

Figure 6. TikTok operation process

\section{ANALYSIS ON ITS ALGORITHMS AND FUNCTIONS}

Like most software today, TikTok's algorithm is also a recommendation algorithm based on users' habits and favourite content. According to the users' interest, comments, and playback completeness, TikTok will intelligently recommend similar videos to users, such as food, news, comedy, etc. It will even recommend relevant product advertisements to you based on the videos you have recently viewed, during the time you browse the videos. At the same time, the TikTok search engine will prompt related search terms. The source of these terms is not only related to the user's usage habits, but also related to the keywords the user has searched recently. TikTok has also established hot lists and celebrity lists. Users can receive the hot trends on TikTok in real time, so that more users can participate in the interaction. Instagram and TikTok can also take short videos, but on TikTok users can use more magic props or video stickers to take videos, which is more interesting than Instagram. Videos uploaded by Instagram can only be viewed in the user's feed, while TikTok can establish instant messaging with friends, share videos and communicate with each other. It can be said that TikTok includes many functions of Instagram, YouTube and Twitter.

Compared with video software like YouTube, in addition to the difference in video duration, YouTube's recommendation mechanism is that man actively accept, while TikTok is passively accepted by you. It means that YouTube is for you to search for what you want to see, and TikTok is for pushing videos based on your favourites. TikTok's brainwashing video push function makes it easy for many users to immerse in. In the world of TikTok you will feel that time passes quickly. This is why more and more people like to use TikTok to spend their time. TikTok can give them the sensory stimulation they want in a short time, while YouTube needs more time. If you want to find entertainment on YouTube, you need time to search, and you can just open TikTok to meet your needs. In this era when time is flooded with all kinds of information, TikTok has successfully occupied your fragmented time. This is the magic of TikTok and the magic of TikTok algorithm.

\section{SURVEY}

In order to get a closer look of TikTok's audience and usage experience, and to understand the public's understanding and acceptance of TikTok's business profit 
model, the author published a questionnaire on the Internet. Here are the questions included in the questionnaire:

Question 1: Do you use TikTok? Please fill in your gender and age.

Question 2: How long do you spend on TikTok every day? Do you have consumer behaviour on TikTok? If so, where did you spend the most? Do you think watching TikTok has become a habit in your life?

Question 3: When you are bored, which is your prior choice, TikTok, social media or video software?

In this questionnaire survey, the author received a total of 2080 valid questionnaires, among which 1810 people reported that they use TikTok, making up $87 \%$ of the total number, indicating that TikTok has a certain mass base and popularity. According to statistics, the proportions of boys and girls who participated in the questionnaire survey were $48 \%$ and $52 \%$, respectively. $12.8 \%$ were under 18 years old, $54.6 \%$ were $18-25$ years old, $26.5 \%$ were $25-35$ years old, and $6.1 \%$ were over 35 years old. According to the data feedback from the first question, TikTok's user gender is basically 50-50, and its main user group is the people between 18 and 35 years old, indicating that young people are abler to accept the products of the new era.

Talking about the second question: How much time do you use TikTok every day? $88 \%$ of people choose within 30 minutes, $9 \%$ choose within one hour, and the remaining $3 \%$ spend on TikTok every day. The time is more than 1 hour. This shows that most people use TikTok for a controlled period of time, and there is basically no over-indulge. Regarding the question "Do you have consumer behaviour on TikTok? If so, where did you spend the most?", 815 respondents had spent on TikTok, accounting for $45 \%$ of the respondents using TikTok. Of these 815 people, $74 \%$ are women, and they spend the most money on TikTok. These data show that TikTok's business model is more attractive to women than men, and women are the most important consumer group. TikTok's business profit model is successful, and it has successfully allowed a considerable number of users to pay. This also shows that short video sales are easier to generate consumer desires than traditional sales methods. Regarding the fourth question, $77 \%$ of the respondents said that watching TikTok became a habit of their lives, which surprised me at the dependence and love of TikTok users who has used TikTok. As for the last question, when you are bored, what kind of software do you choose to spend? $48 \%$ of people choose TikTok, $27 \%$ choose instant messaging software like WeChat and Facebook, and $15 \%$ choose social media like Instagram, Twitter and Weibo, $10 \%$ of people choose video software. These data fully show that when facing fragmented boring time, contemporary people are gradually inclined to use TikTok software that can gain happiness in a short time, thus saving the boring time of waiting for others to reply and search. All of this is recommended based on TikTok's strong algorithmic foundation and mature business operation model, which has allowed TikTok to harvest a large number of loyal users.

\section{CONCLUSION}

According to the research results, it is TikTok's powerful recommendation mechanism algorithm that makes users like this application, and provides accurate promotion for commercial profit activities. Its rich functions also increase the user experience and provide a variety of possible opportunities for commercial profitable activities, such as live broadcast and video placement advertising. The survey found that TikTok's business profit model has a good penetration rate, and many young people like to watch TikTok and consume on it. TikTok has accumulated a huge number of users through short videos, and then turned the flow into cash through profit models such as advertising and ecommerce. TikTok created a new social media profit model and achieved great success. TikTok's great success in overseas markets provides experience and templates for Chinese software to enter the world market. In the final analysis, the key to being welcomed by global users is the powerful and excellent business model of the core technology. Enterprises must firmly grasp the development trend of the market and the next trend of Internet technology, and make good use of the resources and policies of all parties. To be successful in the world, excellent technology and business profit model are indispensable.

\section{ACKNOWLEDGMENT}

Thank you, Professor Steve who taught me the professional knowledge of business analysis, and thank you for your careful explanation and help in the professional class. Thanks to my teaching assistant Yuhui, thank you for helping us sort out our knowledge. Thanks to the students who discussed and made progress with me during the course. Thanks to my thesis advisor Yvonne. Without the help of all of you, I think I might not have realized so much knowledge of business analysis. Thank you all from the bottom of my heart.

\section{REFERENCES}

[1] QuestMobile Research Institute, QuestMobile Short Video 2019 Semi-Annual Report, 2019. https://www.questmobile.com.cn/research/reportnew/58

[2] Cross-border diary. Tiktok/Douyin global user statistics collection. 2020. https://www.cifnews.com/article/68886 
[3] Sensor Tower. The world's top 10 popular mobile app downloads and revenue in July 2020. https://sensortower-china.com

[4] Q. Yu. Investigation Report on the Commercial Value and Profit Model of Douyin Short Video, Nanjing University, 2019

[5] Douyin. Douyin Short Video Marketing Case Manual, https://www.sohu.com/a/252148380_665157 